\section{Klippel-Feil Syndrome and Anterior Cervical \\ Meningomyelocele: A Rare Case Report}

We report a case of Klippel-Feil syndrome associated with an anterior cervical meningomyelocele.

A 36-year old female patient was referred for evaluation of bilateral symmetric small muscle wasting in both hands. MR images (Fig 1) showed fusion of cervical vertebrae and neural arches. There was an anterior meningomyelocele at the level of C6 and C7. The cervical spinal cord was kinked, made an anterior loop, and was dragged anteriorly within the widened posterior thecal sac, stretching the nerve roots within the thecal sac. Part of spinal cord (Fig 2) was lying within the spinal canal and part of it was lying outside the anterior vertebral bodies at the level of C6.

The Klippel-Feil deformity is a complex of osseous and visceral anomalies, which include low hairline, platybasia, fused cervical vertebrae with a short neck, and deafness. Associated central nervous system abnormalities include occipital cephalocele, Chiari I malformation, syrinx, microcephaly, and hydrocephalus. A meningomyelocele is a herniation of the meninges along with the cord through a foramina or defect in the vertebral column and is frequently (> 80\%) located posteriorly in the lumbosacral area. An anterior meningomyelocele is rare and is generally described in the thoracic or sacral region.

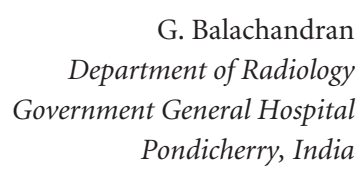

DOI 10.3174/ajnr.A1730

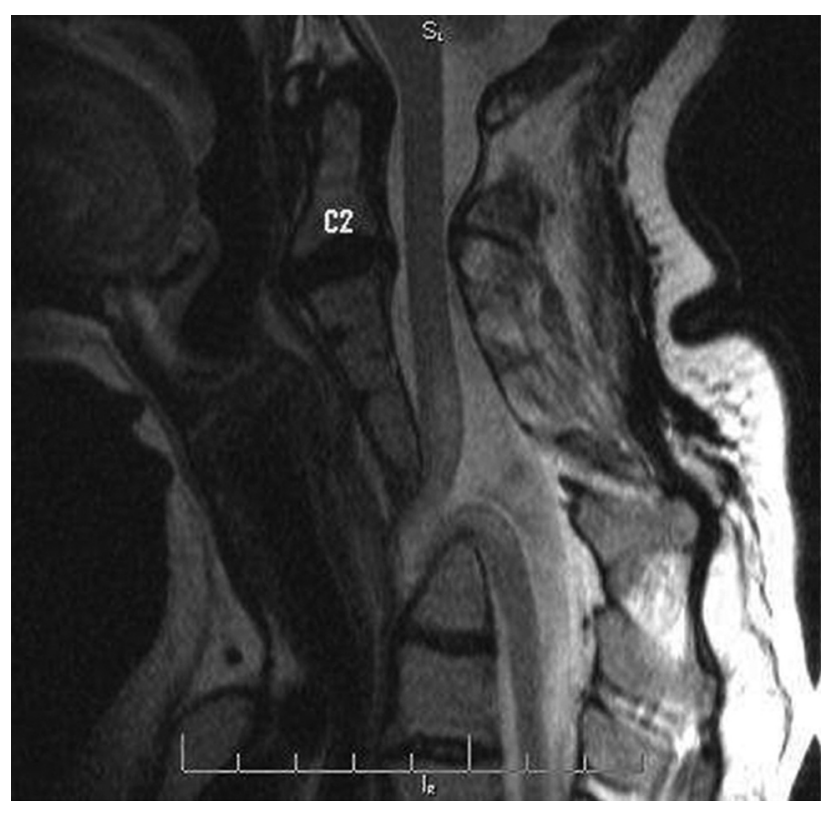

Fig 1. T2-weighted sagittal view of the cervical spine shows fused cervical vertebrae and an anterior meningomyelocele.

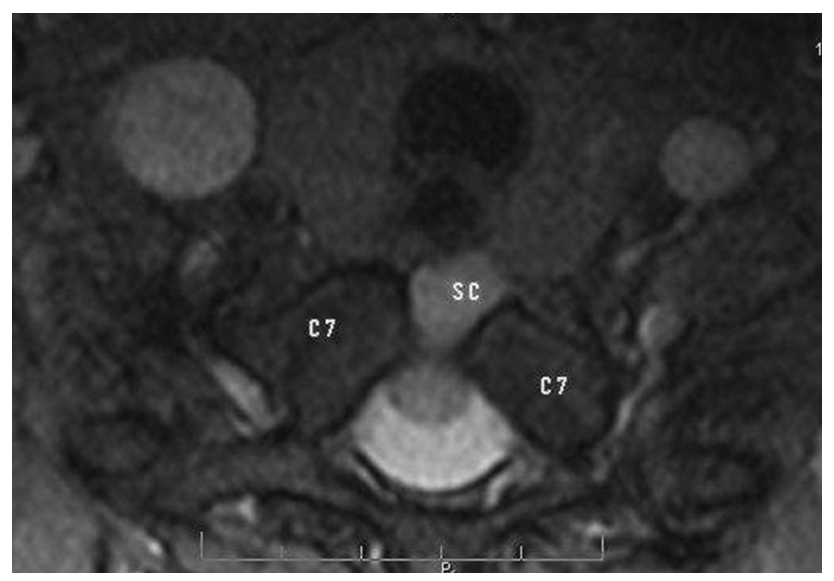

Fig 2. T2-weighted transverse view of the cervical spine shows herniation of spinal cord (SC) through an anterior defect in the $\mathrm{C} 7$ body. 